BULL. AUSTRAL. MATH. SOC.

VOL. 30 (1984), 251-269.

\title{
NEMITSKY OPERATORS BETWEEN \\ ORLICZ-SOBOLEV SPACES
}

\author{
Grahamie HARDY
}

\begin{abstract}
A theorem on Nemitsky operators between Sobolev spaces, contained in a 1973 paper of Marcus and Mizel, is extended to orlicz-Sobolev spaces.
\end{abstract}

\section{Introduction}

Let $\Omega$ be a domain in $R_{n}$, and let the function $g(x, t)$ be defined for $x \in \Omega, t \in \mathrm{R}_{m}$. The Nemitsky operator $G$ is defined on functions $u: \Omega \rightarrow R_{m}$ by

$$
(G u)(x)=g\left(x, u_{1}(x), \ldots, u_{m}(x)\right) \text { for } x \in \Omega
$$

The two papers [6] and [7] of Marcus and Mizel contain theorems giving conditions under which a Nemitsky operator $G$ is a mapping between Sobolev spaces. One of these theorems, Theorem 2.1 in $[7]$, is extended to orlicz-Sobolev spaces in Hardy [4]. In the present paper, we extend another theorem of the same type, Theorem 2.2 in $[6]$.

Part of the proof of this theorem involves estimates of the terms of a chain rule for $G u$. Marcus and Mizel derive a suitable chain rule (see Lemma 2.1 and Theorem 2.1 in [6], and also Equation 3.23 below) under the

Received 9 April 1984. The author wishes to thank Dr. H.B. Thompson for many useful discussions and suggestions made throughout the preparation of this paper. The author also wishes to thank Professor Robert Adams for comments on a preliminary version of this paper. $\$ A 2.00+0.00$.

Copyright Clearance Centre, Inc. Serial-fee code: 0004-9727/84 
assumptions that (i) $g$ is locally Lipschitz on $\Omega \times R_{m}$, and (ii) $g$ possesses a total differential except for a certain null set. (As the complete statement of (ii) is rather long, and moreover is not of direct concern to us, we refer the reader to [6] for details.)

For brevity, we shall refer to conditions (i) and (ii) of Lemma 2.1 in [6] (i.e., (i) and (ii) above) collectively as condition (*).

For $g$ and $\Omega$ as described above, Theorem 2.2 in [6] can then be stated as follows:

THEOREM 1.1. (Marcus and Mizel) Let $\Omega \subset \mathrm{R}_{n}$ be a bounded domain satisfying the cone condition, and suppose $g$ satisfies the condition (*).

Let $\nu \geq 1,1<p<n, r=v m p /(v-1)(n-p),[r=\infty$ for $v=1]$, $q=\mathrm{ump} /[n+(v-1) p]$, and $|t|=\left|t_{1}\right|+\ldots+\left|t_{n}\right|$.

Suppose that, for every $(x, t) \in \Omega \times \mathrm{R}_{m}$ where any derivative mentioned below exists,

$$
\begin{aligned}
& \left|\partial g(x, t) / \partial x_{i}\right| \leq a_{1}(x)+b_{1}|t|^{\nu}, \quad i=1, \ldots, n, \\
& \left|\partial g(x, t) / \partial t_{j}\right| \leq a_{2}(x)+b_{2}|t|^{\nu-1}, \quad j=1, \ldots, m,
\end{aligned}
$$

where $b_{1}$ and $b_{2}$ are constants, $a_{1} \in L_{p}(\Omega)$, and $a_{2} \in L_{p}(\Omega)$.

Then

$$
G: W_{1, q^{(\Omega)^{m}}+W_{1, p}(\Omega)}
$$

and

$$
\text { (1.5) }\|G u\|_{W_{1, p}}(\Omega) \leq \operatorname{const}\left(1+\|u\|_{W_{1, q}}^{\nu}(\Omega)^{m)} \text { for alz } u \in W_{1, q}(\Omega)^{m}\right. \text {, }
$$

where the constant depends on $\Omega, a_{1}, a_{2}, b_{1}, b_{2}$, and $g(x, 0)=f(x)$, but not on $n$.

For our extension of this theorem (see Theorem 3.1 below), we also assume that $g$ satisfies condition $(*)$, but make the following changes to the bounds given in (1.2) and (1.3): 
Nemitsky operators between Orlicz-Sobolev spaces

(a) the power function $|t|^{\nu}$ is replaced by an $N$-function $M$, assumed to satisfy a $\Delta_{2}$-condition, and to have an increasing derivative;

(B) the Lebesgue spaces $L_{p}, L_{q}, L_{r}$ are replaced by suitable orlicz spaces $L_{\mathbf{P}}, L_{\mathrm{Q}}$, and $L_{\mathbf{R}^{*}}$

We then find that, with the Sobolev spaces replaced by OrliczSobolev spaces, the conclusion (1.4) still holds. We also obtain an estimate analogous to (1.5).

We conclude with two examples. The first, Example 4.1, shows that our Theorem 3.1 contains Theorem 1.1 (for $\nu>1$ only) as a particular case. The second, Example 4.2, shows that our Theorem 3.1 contains results not included in Theorem 1.1 .

\section{Preliminaries}

2.1. ORLICZ SPACES. We shall use the properties of $N$-functions and Orlicz spaces as given in Krasnosel'ski $\dot{i}$ and Ruticki $\dot{i}$ [5]. In some places, all of which occur in the list of facts below, our notation differs from that in [5], and sometimes, for later convenience, we have introduced a more specific notation. We shall only need to consider Orlicz spaces defined on bounded domains $\Omega \subset R_{n}$.

Let $M$ be an $N$-function, and let $M_{+}^{\prime}$ denote the right derivative of $M$. Then

(i) $\quad u M_{+}^{\prime}(u)>M(u)$ for $u>0$,

and

(ii) $M(\alpha u) \leq \alpha M(u)$ for $0 \leq \alpha \leq 1$.

The inequality is reversed if $\alpha \geq 1$.

$M$ is said to satisfy the $\Delta_{2}$-condition if there exists a constant $u_{0} \geq 0$ and a constant $(\geq 2)$, here denoted by $K_{M}$, such that

(iii) $M(2 u) \leq K_{M}(u)$ for $u \geq u_{0}$.

A condition equivalent to (iii) is that there exists a real-valued 
function, here denoted by $k_{M}$, and a constant $u_{0} \geq 0$, such that, for $\ell>1$,

(iv) $M(\ell u) \leq k_{M}(\ell) M(u)$ for $u \geq u_{0}$.

From (ii), $k_{M}(\ell) \geq 1$, and a modification of the proof of (iv) given in [5] shows that $k_{M}$ may be chosen to have the form $k_{M}(\ell)=C \ell^{\mu}$, where $C>1$ and $\mu>1$.

Suppose $\Psi$ is a function which is right-continuous, non-decreasing, and positive, for positive values of its argument (for example, $M_{+}^{\prime}$ ), and for which an inequality of the form (iii) holds. We shall still say that $\Psi$ satisfies the $\Delta_{2}$-condition, even if $\Psi$ is not an $N$-function.

Conditions (iii) and (iv) are still equivalent, but now we do not necessarily have $k_{\Psi}(\ell) \geq 1$ for $\ell>1$.

If $M$ satisfies the $\Delta_{2}$-condition, there exists a constant $u_{0}>0$ such that

(v) $\quad u M^{\prime}(u) / M(u)<K_{M}$ for all $u \geq u_{0}$.

(vi) If the function $Q$ is convex and even for large values of $u$ and if $\lim Q(u) / u=\infty$, then there exists an $N$-function $M$ such that $u \rightarrow \infty$

$M(u)=Q(u)$ for large values of $u$, and in this case $Q$ is said to be the principal part of $M$.

(vii) If $M$ is an $N$-function, we may characterise the orlicz space $L_{M}=L_{M}(\Omega)$ as the set of all measurable functions $u$, defined almost everywhere on $\Omega$, such that there exists a constant $\lambda>0$ such that $\int_{\Omega} M[\lambda u(x)] d x<\infty$.

We shall use the Luxemburg norm, here denoted by $\|\cdot\|_{M}=\|\cdot\|_{M, \Omega}$. (viii) If $\|u\|_{M}>0, \int_{\Omega} M\left[u(x) /\|u\|_{M}\right] d x \leq 1$.

(ix) Let $M_{1}$ and $M_{2}$ be $N$-functions. If there exist constants $u_{0}$ and $K$ such that $M_{2}(u) \leq M_{1}(K u)$ for $u \geq u_{0}$, we write $M_{2}<M_{1}$, and 
Nemitsky operators between orlicz-Sobolev spaces

in this case there exists a constant $C$ such that

$$
\|u\|_{2} \leq C\|u\|_{1} \quad \text { for } u \in L_{M_{1}} \text {. }
$$

(x) Let $P, Q$ and $R$ be $N$-functions, and suppose that there exist complementary $N$-functions $\Phi$ and $\tilde{\hat{\varphi}}$ such that

$$
\Phi<P^{-1} \circ Q
$$

and

$$
\tilde{\Phi}<P^{-1} \circ R
$$

Then there exists a constant $C$ such that

$$
\|z a w\|_{P} \leq C\|u\|_{Q}\|w\|_{R} \text {. }
$$

(The notation " $<$ " is still meaningful even if $P^{-1} \circ Q$ and $P^{-1} \circ R$ are not $N$-functions.)

Here, and elsewhere in this paper, we shall use the convention made on page 8 of [5] to define the inverse of an $\mathrm{N}$-function. (The convention is also made that $P^{-1}(v)$ denotes $\left.P^{-1}(|v|).\right)$

2.2. ORLICZ-SOBOLEV SPACES. We shall use the definitions and properties of Orlicz-Sobolev spaces as given in Donaldson and Trudinger [2]. See also Adams [1]. We shall only need to consider these spaces defined on bounded domains $\Omega \subset R_{n}$ satisfying the cone condition.

Let $Q$ be an $N$-function. Then the Sobolev conjugate $N$-function $Q_{*}$ of $Q$ is defined by

$$
Q_{*}^{-1}(s)=\int_{0}^{|s|} Q^{-1}(t) \cdot t^{-1-1 / n} d t
$$

where it is assumed that, if necessary, $Q(t)$ is redefined for small values of $t$ (obtaining an equivalent $l /$-function) so that

$$
\int_{0}^{1} Q^{-1}(t) \cdot t^{-1-1 / n} d t<\infty .
$$

From the stronger result $Q \ll Q_{*}$, proved in Gossez [3], we have

$$
Q<Q_{*}
$$




\section{Grahame Hardy}

The Orlicz-Sobolev space $W^{1} L_{Q}(\Omega)$ is defined as the set of all functions in $L_{Q}(\Omega)$ whose distributional derivatives $\partial_{i} u$ also belong to $L_{Q}(\Omega)$. A norm $\|u\|_{1, Q}=\|u\|_{1, Q, \Omega}$ may be defined on $W^{1} L_{Q}(\Omega)$ by

(iv)

$$
\| u{ }_{1, Q}=\max \left\{\|u\|_{Q}\left\|\partial_{1} u\right\|_{Q}, \ldots,\left\|\partial_{n} u\right\|_{Q}\right\} .
$$

In the case that $\lim _{s \rightarrow \infty} Q_{*}^{-1}(s)=\infty$, there exists a constant $C$ such that

$$
\|\|_{Q}\left\|_{*} \leq C\right\| u \|_{1, Q, \Omega}
$$

We shall denote the set of vector-valued functions $u=\left(u_{1}, \ldots, u_{m}\right)$, each of whose components belong to $W^{1} L_{Q}(\Omega)$, by $W^{1} L_{Q}(\Omega)^{m}$, and use the norm

$$
\|u\| 1, Q, \Omega^{m}=\max _{1 \leq j \leq m}\left\|u_{j}\right\| 1, Q, \Omega
$$

on this space.

Following [6], let $A(\Omega)$ denote the set of all functions $u$, measurable on $\Omega$, such that for almost all lines $\tau$ parallel to any coordinate axis $x_{i}, i=1, \ldots, n, u$ is locally absolutely continuous on $\tau \cap \Omega$. The orlicz-Sobolev spaces $W^{1} L_{P}(\Omega)$ may be given an alternative characterisation in terms of the class $A(\Omega)$, as follows:

(vii) Let $\Omega$ be a bounded domain in $\mathrm{R}_{n}$ with the cone property, and let $P$ be an $N$-function. A function $u$ defined on $\Omega$ is in ${ }^{1} L_{P}(\Omega)$ if and only if there exists $\tilde{u} \in A(\Omega)$ such that

(a) $\tilde{u}=u$ almost everywhere in $\Omega$;

(B) $\partial \tilde{u} / \partial x_{i} \in L_{P}(\Omega), \quad(i=1, \ldots, n)$.

Further $\partial \tilde{u} / \partial x_{i}=\partial_{i} u$ almost everywhere in $\Omega$.

(vii) is an immediate consequence of Lemma 1.5 in [6] and Theorem 3.1 in Hardy [4]. 


\section{A theorem on Nemitsky operators}

We need the three lemmas which follow. The notation introduced in $\$ 2$ will be used without further comment.

LEMMA 3.1. Suppose the $N$-function $M$ satisfies the $\Delta_{2}$-condition, so that, for large enough $u$,

$$
M(\ell u) \leq k_{M}(\ell) M(u) \text { for } \ell>1 \text {. }
$$

Then

$$
M_{+}^{\prime} \text { satisfies the } \Delta_{2} \text {-condition, }
$$

and further, there exists a constant $C$ such that, for large enough $u>0$,

(iii)

$$
M_{+}^{\prime}(\ell u) \leq\left[C k_{M}(\ell) / \ell\right] M(u) \text { for } \ell>1 \text {. }
$$

(Thus we may take $k_{M_{+}^{\prime}}(\ell)$ as $\left.C k_{M}(\ell) / \ell.\right)$

Proof, From 2.1(v), 2.1(iii), and 2.1(i), we have, for sufficiently large $u>0$,

$$
M_{+}^{\prime}(2 u)<K_{M} M(2 u) /(2 u) \leq K_{M}^{2} M(u) /(2 u) \leq\left(K_{M}^{2} / 2\right) M_{+}^{\prime}(u),
$$

which proves (ii).

For $\ell>1$ and sufficiently large $u>0$,

$$
\ell \int_{0}^{u} M_{+}^{\prime}(\ell t) d t=M(\ell u) \leq k_{M}(\ell) M(u)=k_{M}(\ell) \int_{0}^{u} M_{+}^{\prime}(t) d t
$$

so

$$
(\ell u / 2) M_{+}^{\prime}(\ell u / 2) \leq k_{M}(\ell) u M_{+}^{\prime}(u),
$$

and then, for large enough $w=u / 2$,

$$
M_{+}^{\prime}(\ell w) \leq\left[2 k_{M}(\ell) / \ell\right] M_{+}^{\prime}(2 w) \leq 2 K_{M_{+}^{\prime}}\left[k_{M}(\ell) / \ell\right] M_{+}^{\prime}(w)
$$

on using (ii). This proves (iii).

Note. In our applications of the next lemma, $\Psi$ will be either an $N-$ function $M$ or its derivative $M^{\prime}$, where it is supposed that $M^{\prime}$ exists and is strictly increasing. To avoid trivial complications, we shall make 
the convention that $M^{\prime}(u)$ denotes $M^{\prime}(|u|)$ if $u<0$, and that the conventions of $2.1(x)$ apply to $\Psi$.

LENAA 3.2. Let $P$ be an N-function, and let $\Psi(v)$ be an even function which is continuous and strictly increasing for all $v \geq 0$, and such that $\Psi(0)=0$ and $\Psi(v) \rightarrow \infty$ as $v \rightarrow \infty$. Suppose further that $P \circ \Psi^{-1}$ is an $N$-function.

Then, if $\Omega$ is a bounded domain in $R_{n}$,

(i) $u \in L_{P}(\Omega)$ implies that $\Psi(u) \in L_{p_{0} \Psi-1}(\Omega)$ if and only if $\Psi$ satisfies that " $\Delta_{2}$-condition;

(ii) in the case that $\Psi$ satisfies the $\Delta_{2}$-condition, there exists a constant $C$ such that

$$
\|\Psi(u)\|_{P_{0} \Psi-1} \leq C\left[1+k_{\Psi}\left(\|u\|_{P}\right)\right],
$$

where the function $k_{\Psi}$ is as in 2.1(iv).

Proof. Suppose first that $\Psi$ satisfies the $\Delta_{2}$-condition. Since (3.1) is obvious if $\|u\|_{P}=0$, we have only to consider the cases (A) and (B) below.

$$
0<\|u\|_{P, \Omega} \leq 1
$$

Then

$$
\int_{\Omega} P[u(x)] d x \leq \int_{\Omega} P[u(x) /\|u\|] P^{]} d x \leq 1,
$$

using 2.1 (viii), and so

$$
1 \in\left\{\lambda>0: \int_{\Omega}\left(P \circ \Psi^{-1}\right)[\Psi(u(x)) / \lambda] d x \leq 1\right\},
$$

and since this implies that $\|\Psi(u)\|_{P \circ \Psi^{-1}} \leq 1,(3.1)$ follows in this case.

(B)

$$
1<\left\|_{u}\right\|_{P, \Omega}<\infty
$$

Let $u_{0}$ and $k=k_{\Psi}$ be as in 2.1 (iv). Let 
Nemitsky operators between Orlicz-Sobolev spaces

$$
\Omega_{0}=\left\{x \in \Omega:|u(x)|<\| u{ }_{P} u_{0}\right\}
$$

and let

$$
\Omega_{1}=\Omega \backslash \Omega_{0}
$$

For $x \in \Omega_{0}$, because $\Psi(v)$ increases with $v$,

$$
\left.\Psi r_{-} u(x)\right] \leq \Psi\left(\|u\|_{P} u_{0}\right) \leq k\left(\|u\|_{P}\right) \Psi\left(u_{0}\right)
$$

and then, (denoting Lebesgue measure by $|\cdot|$ )

$$
\int_{\Omega_{0}}\left(P \circ \Psi^{-1}\right)\left\{\Psi\lceil\cdot u(x)] / k\left(\|u\|_{P}\right)\right\} d x \leq\left|\Omega_{0}\right| P\left(u_{0}\right)
$$

For $x \in \Omega_{1}$, since then $|u(x)| /\|u\|_{P} \geq u_{0}$,

$$
\Psi[u(x)]=\Psi\left[\|u\|_{P} \cdot u(x) /\|u\|_{P}\right] \leq k\left(\|u\|_{P}\right) \Psi\left[u(x) /\left\|_{u}\right\|_{P}\right]
$$

and so

$$
\int_{\Omega_{1}}\left(P \circ \Psi^{-1}\right)\left\{\Psi[u(x)] / k\left(\|u\|_{P}\right)\right\} d x \leq \int_{\Omega_{1}}\left(P \circ \Psi^{-1}\right)\left\{\Psi\left[u(x) /\left\|_{u}\right\|_{P}\right]\right\} d x
$$

$$
\leq \int_{\Omega} P\left[u(x) /\|u\|_{P}\right] d x \leq 1
$$

Adding the inequalities (3.2) and (3.3) and using 2.l(ii), we have

$$
\left.\int_{\Omega}\left(P \circ \Psi^{-1}\right)\left\{\Psi[u(x)] /\left[1+\left|\Omega_{0}\right| P\left(u_{0}\right)\right] k\left(\left\|_{u}\right\|_{P}\right)\right]\right\} d x \leq 1,
$$

which completes the proof of (3.1), from the definition of the Luxemburg norm.

Now suppose that $\Psi$ does not satisfy the $\Delta_{2}$-condition. Then there exists a positive increasing unbounded sequence $\left\{u_{j}\right\}$ such that

$$
\Psi\left(2 u_{j}\right)>2^{2 j_{\Psi}}\left(u_{j}\right)
$$




\section{Grahame Hardy}

We may divide $\Omega$ into disjoint measurable sets $\Omega_{j}$ such that

$$
\left|\Omega_{j}\right|=P\left(u_{1}\right)|\Omega| /\left\lceil 2^{j} P\left(u_{j}\right)\right\rceil, \quad j=1,2, \ldots .
$$

We now define $u: \Omega \rightarrow R$ by

$$
u(x)= \begin{cases}2 u_{j} & \text { for } x \in \Omega_{j}, j=1,2, \ldots, \\ 0 & \text { otherwise in } \Omega,\end{cases}
$$

and then, on using (3.4), we find that

$$
\int_{\Omega} P[u(x) / 2] d x=\sum_{j=1}^{\infty} P\left(u_{j}\right)\left|\Omega_{j}\right|<\infty .
$$

Therefore $u / 2$ and hence $u \in L_{p}(\Omega)$.

Let $\lambda>0$, and let $m$ be a positive integer such that $\lambda>1 / 2^{m}$. Then

$$
\begin{aligned}
\int_{\Omega}\left(P \circ \Psi^{-1}\right)\{\lambda \Psi[u(x)]\} d x & =\sum_{j=1}^{\infty}\left|\Omega_{j}\right|\left(P \circ \Psi^{-1}\right)\left[\lambda \Psi\left(2 u_{j}\right)\right] \\
& >\sum_{j=1}^{\infty}\left|\Omega_{j}\right|\left(P \circ \Psi^{-1}\right)\left[\lambda 2^{2 j} \Psi\left(u_{j}\right)\right] \\
& >\sum_{j=m}^{\infty}\left|\Omega_{j}\right|\left(P \circ \Psi^{-1}\right)\left[2^{j} \Psi\left(u_{j}\right)\right] \\
& \geq \sum_{j=m}^{\infty}\left|\Omega_{j}\right| 2^{j}\left(P \circ \Psi^{-1}\right)\left[\Psi\left(u_{j}\right)\right]=\infty,
\end{aligned}
$$

where we have used $(3.4), 2.1(i i)$, and the assumption that $P \circ \Psi^{-1}$ is an $N$-function. From 2.1(vii), $\Psi(u) \notin L_{P} \circ \Psi-1$, and the proof is complete.

REMARK. Suppose the function $\Psi$ in Lemma 3.2 is also an $N$-function satisfying the $\Delta^{\prime}$-condition, i.e., there exist constants $c>0$ and $u_{0}>0$ such that

$$
\Psi(u v) \leq c \Psi(u) \Psi(v) \text { for } u, v \geq u_{0}
$$


Nemitsky operators between Orlicz-Sobolev spaces

Without loss of generality, we may assume that $u_{0} \geq 1$. Then if $1<\ell \leq u_{0} \leq u$,

$$
\Psi(\ell u) \leq \Psi\left(u_{0} u\right) \leq c \Psi\left(u_{0}\right) \Psi(u) \leq c\left[\Psi\left(u_{0}\right) / \Psi(1)\right] \Psi(\ell) \Psi(u) ;
$$

and since the case in which $\ell>u_{0}$ follows directly from (3.5), we have

$$
\Psi(\ell u) \leq c_{1} \Psi(\ell) \Psi(u) \text { for } \ell>1, u \geq u_{0} \text {, }
$$

where $c_{1}=c \max \left\{1, \Psi\left(u_{0}\right) / \Psi(1)\right\}$. Thus we may take $k_{\Psi}=c_{1} \Psi(\ell)$, so that the estimate (3.1) takes the form

$$
\|\Psi(u)\|_{P \circ \Psi-1} \leq \operatorname{const}\left[1+\Psi\left(\|u\|_{P}\right)\right] .
$$

LEMMA 3.3. Suppose $M, P$, and $Q$ are $N$-functions satisfying the following conditions:

(i) For all positive values of $u, M^{\prime}(u)$ is strictly increasing and. continuous:

(ii) For $Q_{*}$ as in 2.2(i), and for Zarge $v$,

$$
P^{-1}(v)=M^{\prime} \circ Q_{*}^{-1}(v) \cdot Q^{-1}(v) ;
$$

(iii) $R=Q_{*}{ }^{\circ}\left(M^{\prime}\right)^{-1}$ is an $N$-function.

Then

(iv) $Q_{*} \circ M^{-1}$ is the principal part of an $N$-function,

$$
Q_{*} \circ M^{-1} \sim P_{*},
$$

and, if $\Omega$ is a bounded domain in $R_{n}$, there exists a constant $C$ such that

$$
\left\|f_{1} f_{2}\right\|_{P, \Omega} \leq C f_{1}\left\|_{R, \Omega}\right\| f_{2} \|_{Q, \Omega}
$$

whenever $f_{1} \in L_{R, \Omega}$ and $f_{2} \in L_{Q, \Omega}$.

Proof. From 2.2(i) and (3.7), we have

$$
\left(M \circ Q_{*}^{-1}\right) \prime(v)=\left(P_{*}^{-1}\right) \cdot(v)
$$

for large enough $v$. Using (3.10) and the fact that $P_{*}$ is an 


\section{Grahame Hardy}

$N$-function, we then find that $M \circ Q_{*}^{-1}(v)$ is concave for large enough $v$, and that $M \circ Q_{*}^{-1}(v) / v \rightarrow 0$ as $v \rightarrow \infty$, which shows that $Q_{*} \circ M^{-1}$ is the principal part of an $N$-function. On using Lemma 3.2 in [5] as well, we obtain (3.8).

Substituting $v=Q(u)$ in (3.7) we obtain, for some $U_{1} \geq 0$,

$$
\begin{aligned}
\left(P^{-1} \circ Q\right)(u) & =u \cdot\left(M^{\prime} \circ Q_{*}^{-1} \circ Q\right)(u) \quad u \geq U_{1} \\
& =u \phi(u),
\end{aligned}
$$

where $\phi$, defined by

$$
\phi(u)=\left(M^{\prime} \circ Q_{*}^{-1} \circ Q\right)(u)
$$

is continuous and increasing for $u \geq U_{1}$. For any $U \geq U_{1}$ and $u>U$,

$$
\int_{U}^{u} \phi(t) d t \leq \phi(u)(u-U) \leq u \phi(u)-U \phi(U) \text {, }
$$

and so

$$
\left(P^{-1} \circ Q\right)(u) \geq\left(P^{-1} \circ Q\right)(U)+\int_{U}^{u} \phi(t) d t .
$$

From (3.8) and (3.11), for sufficiently large values of the argument,

$$
P^{-1} \circ R=\left(M^{\prime} \circ Q_{*}^{-1} \circ R\right) \cdot Q^{-1} \circ R=\left[M^{\prime} \circ Q_{*}^{-1} \circ Q_{*} \circ\left(M^{\prime}\right)^{-1}\right] \cdot Q^{-1} \circ Q_{*} \circ\left(M^{\prime}\right)^{-1}
$$

and therefore there exists $U_{2} \geq 0$ such that

$$
\left(P^{-1} \circ R\right)(u)=u \phi^{-1}(u) \text { for } u \geq U_{2} \text {. }
$$

Therefore, for any $u \geq U_{2}$, we have

$$
\left(P^{-1} \circ R\right)(U) \geq\left(P^{-1} \circ R\right)(U)+\int_{U}^{U} \phi^{-1}(s) d s .
$$

From [5], pages 16 and 17, $U$ may be chosen large enough so that there exists an $N$-function $\Phi$ such that

$$
\Phi(u)=\left(P^{-1} \circ Q\right)(U)+\int_{U}^{u} \phi(t) d t, \quad u \geq U .
$$




\section{Nemitsky operators between Orlicz-Sobolev spaces}

It is evident from the construction referred to above that the complementary function $\tilde{\Phi}$ to $\Phi$ has the representation

$$
\tilde{\Phi}(u)=K+\int_{U_{3}}^{u} \phi^{-1}(s) d s, \quad u \geq U_{3} \text {, }
$$

where $K>0$ and $U_{3} \geq 0$ are constants. Then if $u_{0}=\max \left\{U_{1}, U_{2}, U_{3}\right\}$ and $0<\beta \leq \min \left\{1, P^{-1}\left[R\left(u_{0}\right)\right] / K\right\}$, we have, for $u \geq u_{0}$,

$$
\begin{aligned}
\tilde{\Phi}(\beta u) \leq \beta \tilde{\Phi}(u) & \leq\left(P^{-1} \circ R\right)\left(u_{0}\right)+\beta \int_{u_{0}}^{u} \phi^{-1}(s) d s \\
& \leq\left(P^{-1} \circ R\right)(u) .
\end{aligned}
$$

By (3.12), (3.14), and (3.15), $\Phi<P^{-1} \circ Q$ and $\tilde{\Phi}<P^{-1} \circ R$, so that (3.9) follows from $2.1(x)$.

We can now obtain our extension of Theorem 1.1. We shall assume that the function $k_{M}$ of 2.1 (iv) is taken to be of the form

$$
k_{M}(\ell)=C \ell^{\mu}, \quad(\ell>1)
$$

where $C>1$ and $\mu>1$ are constants.

THEOREM 3.1. Let $\Omega$ be a bounded domain in $R_{n}$ satisfying the cone condition, and suppose that the function $g$ defined on $\Omega \times R_{m}$ satisfies the condition (*) of Theorem 1.1. Let $M$ and $P$ be N-functions such that

(a) $M$ satisfies the $\Delta_{2}$-condition and $M^{\prime}(u)$ is strictly increasing and continuous for $u>0$;

(B) $P_{*}^{-1}(s) \rightarrow \infty$ as $s \rightarrow \infty$.

Suppose that the $N$-functions $Q$ and $R$ are as in Lemma 3.3 , so that

$$
\begin{aligned}
& P_{*} \sim Q_{*} \circ M^{-1} ; \\
& R=Q_{*} \circ\left(M^{\prime}\right)^{-1},
\end{aligned}
$$




\section{Grahame Hardy}

and suppose that $Q_{*} \circ M^{-1}$, as well as $Q_{*} \circ\left(M^{\prime}\right)^{-1}$, is an $N$-function.

Further, suppose that for every $(x, t) \in \Omega \times R_{m}$ where any derivative mentioned below exists,

$$
\begin{array}{ll}
\left|\partial g(x, t) / \partial x_{i}\right| \leq a_{1}(x)+b_{1} M(|t|) ; & i=1, \ldots, n, \\
\left|\partial g(x, t) / \partial t_{j}\right| \leq a_{2}(x)+b_{2} M^{\prime}(|t|) ; & j=1, \ldots, m,
\end{array}
$$

where $a_{1} \in L_{P}(\Omega), a_{2} \in L_{R}(\Omega)$, and $b_{1}$ and $b_{2}$ are positive constants. Then

$$
G: W^{1} L_{Q}(\Omega)^{m} \rightarrow w^{1} L_{P}(\Omega)
$$

and

$$
\|G u\|_{1, P, \Omega} \leq \text { const }\left[1+k_{M}\left(\|u\|_{\left.\left.1, Q, \Omega^{m}\right)\right]} .\right.\right.
$$

Proof. Since the proof (except for the derivation of the inequalities (3.24), (3.25), and (3.26)) follows that of Theorem 2.2 in Marcus and Mizel [6], it will only be given in outline.

Using 2.2 (vii) (instead of Lemma 1.5 in (6]) we find that $f(x)=g(x, 0) \in W^{1} L_{P}(\Omega)$, and that, if $u \in W^{1} L_{Q}(\Omega)^{m}$ and $v=G u$, the chain rule

$$
\partial_{i} v=\partial g(\cdot, u) / \partial x_{i}+\sum_{j=1}^{m} \partial g(\cdot, u) / \partial t_{j} \partial_{i} u_{j}, \quad i=1, \ldots, n,
$$

holds almost everywhere in $\Omega$. Further, there exists $\tilde{u}_{j} \in A(\Omega)$ such that $\tilde{u}_{j}=u_{j}$ almost everywhere in $\Omega, j=1, \ldots, m$. Let $\tilde{v}=\tilde{G}$. observing that $(3.17)$ and $(\beta)$ implies that $Q_{*}^{-1}(s) \rightarrow \infty$ as $s \rightarrow \infty$, and using Minkowski's inequality, 2.1(ix), 2.2(iii), 2.2(v), Lemma 3.2, and (3.16), we have

$$
\begin{aligned}
& \|M(|\tilde{u}|)\|_{P} \leq \operatorname{const}\|M(|\tilde{u}|)\|_{P_{*}} \leq \operatorname{const}\|M(|\tilde{u}|)\|_{Q_{*}{ }^{\circ} H^{-1}} \\
& \leq \operatorname{const}\left[1+k_{M}\left(\|\tilde{u} \mid\|_{Q_{*}}\right] \leq \mathrm{const}\left[1+k_{M}\left(\|\tilde{u}\|_{1, Q, \Omega^{m}}\right]\right] .\right.
\end{aligned}
$$


Similarly we obtain (using (3.18) and Lemmas 3.1 and 3.3 as well):

$$
\sum_{j=1}^{m}\left\|a_{2} \partial \tilde{u}_{j} / \partial x_{i}\right\|_{P} \leq \text { const } \sum_{j=1}^{m}\left\|a_{2}\right\|_{R}\left\|^{\partial} u_{j} / \partial x_{i}\right\|_{Q}
$$

$$
\leq \text { const }\|\tilde{u}\|, Q, \Omega^{m},
$$

and

$$
\begin{aligned}
& \sum_{j=1}^{m}\left\|M^{\prime}(|\tilde{u}|) \partial \tilde{u}_{j} / \partial x_{i}\right\|_{P} \leq \operatorname{const} \sum_{j=1}^{m}\left\|M^{\prime}(|\tilde{u}|)\right\|_{R}\left\|\partial u_{j} / \partial x_{i}\right\|_{Q} \\
& \leq \text { const }\left[1+k_{M},\left(\||\tilde{u}|\|_{Q_{*}}\right)\right]\|\tilde{u}\|{ }_{1, Q, \Omega^{m}} \\
& \leq \text { const }\left[1+k_{M},\left(\|\tilde{u}\| 1, Q, \Omega^{m}\right)\right]\|\tilde{u}\| 1, Q, \Omega^{m} \\
& \left.\leq \text { const }[\|\tilde{u}\|] 1, Q, \Omega^{m}+k_{M}\left(\|\tilde{u}\| 1, Q, \Omega^{m}\right)\right] .
\end{aligned}
$$

Combining (3.19), (3.20), (3.23), (3.24), (3.25) and (3.26) then gives

$$
\left\|\partial v / \partial x_{i}\right\|_{P} \leq \operatorname{const}\left[1+\|\tilde{u}\|_{1, Q, \Omega^{m}}+k_{M}\left(\|\tilde{u}\|_{1, Q, \Omega}{ }^{m}\right)\right]
$$

$$
\leq \text { const }\left[1+k_{M}\left(\|\tilde{u}\| 1, Q, \Omega^{m)}\right] .\right.
$$

Finally, from the argument in [6] and (3.24), we obtain

$$
\|\tilde{v}-f\|_{P} \leq \text { const }\left[1+k_{M}\left(\|\tilde{u}\|_{\left.\left.1, Q, \Omega^{m}\right)\right]} .\right.\right.
$$

(3.27) and (3.28) give the estimate (3.22).

\section{Examples}

We shall now give two sets of $N$-functions $M, P, Q$, and $R$ satisfying the hypotheses of Theorem 3.1 .

EXAMPLE 4.1. Let $v>1,1<p<n, q=u m p /[n+(v-1) p]$, and $r=v n p /(v-1)(n-p)$, as in Theorem 1.1. Then $1<q<n, 1 / r+1 / q=1 / p$, and if we let $p_{*}=n p /(n-p)$ and $q_{*}=n q /(n-q)$, we have 
$q_{*}=\nu p_{*}=(\nu-1) x$. Let $M(u)=|u|^{\nu}, Q(u)=q_{*}^{q}|u|^{q}$, and $P(u)=p_{*}^{p}|u|^{p}$. From $2.2(i), Q_{*}(u)=|u|^{q_{*}}, P_{*}(u)=|u|^{p_{*}}=|u|^{q_{*} / v}=Q_{*} \circ M^{-1}(u)$, and $P_{*}^{-1}(s) \rightarrow \infty$ as $s \rightarrow \infty$. Let $R(u)=v^{-q_{*} /(v-1)}|u|^{q_{*}(v-1)}=v^{-r}|u|^{p}=$ $Q_{*} \circ\left(M^{\prime}\right)^{-1}(u)$.

For $M(u)$ as above, $k_{M}(\ell)=\ell^{\nu}$, and we obtain Theorem 1.1 (for $\nu>1$ only) as a particular case of Theorem 3.1 .

EXAMPLE 4.2. Let $v, p, r, q$ and $Q$ be as in Example 4.1. For $u_{0}>1$, let

$$
M(u)=|u|^{v}(\ln |u|+1) \quad \text { for } \quad|u| \geq u_{0}
$$

Let

$$
\alpha(u)=u M_{+}^{\prime}(u) / M(u)=v+1 /(\ln u+1)
$$

and let $\alpha_{0}$ denote $\alpha\left(u_{0}\right)$. We complete the definition of $M$ by defining

$$
M(u)=M\left(u_{0}\right)\left(|u| / u_{0}\right)^{\alpha} \text { for } \quad 0 \leq|u| \leq u_{0} \text {. }
$$

From the arguments in [5], pages 16 and 17, $M$ is a differentiable $N$-function which also satisfies the $\Delta_{2}$-condition. Since $\alpha(u)+v$ as $u \rightarrow \infty$ and $v / q_{*}=1 / p_{*}<1$, we may also assume that $u_{0}$ is chosen large enough so that $M \circ Q_{*}^{-1}(v)$ is concave for $0 \leq v \leq u_{0}^{q_{*}}$. Note that this last relation is not disturbed if $u_{0}$ is further enlarged.

For sufficiently large positive $v$, we have

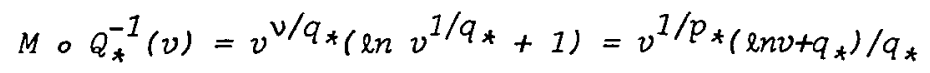

and then

$$
\left.\left(M \circ Q_{*}^{-1}\right) \prime(v)=v^{\left(1 / p_{*}\right)-2}\left[-\left(1-1 / p_{*}\right)\left(\ln v+q_{*}+p_{*}\right)+1\right)\right] / p_{*} q_{*},
$$

which is negative for sufficiently large $v$. Thus we may enlarge $u_{0}$ so that $M \circ Q_{*}^{-1}$ is concave for all positive values of the argument, i.e., (i) $Q_{*} \circ M^{-1}$ is convex.

The limit 
Nemitsky operators between Orlicz-Sobolev spaces

(ii) $\lim _{u \rightarrow 0+} Q_{*} \circ M^{-1}(u) / u=0$

follows from $(4.2)$ and the fact that $q_{*} / \alpha_{0}$ is greater than 1 . The limit

(iii) $\lim _{u \rightarrow \infty} Q_{*} \circ M^{-1}(u) / u=\infty$

follows from the equivalent limit

$$
\lim _{v \rightarrow \infty} M \circ Q_{*}^{-1}(v) / v=0 \text {, }
$$

obtained from (4.3). From (i), (ii), and (iii), it follows that $Q_{*} \circ M^{-1}$ is an $N$-function.

Similar arguments, using the fact that $q_{*} /(\nu-1)=r>p_{*}>1$, show that $R=Q_{*}{ }^{\circ}\left(M^{\prime}\right)^{-1}$ is an $N$-function.

For large positive values of the argument, we define $P$ by

$$
\begin{aligned}
P^{-1}(v) & =M^{\prime} \circ\left[Q_{*}^{-1}(v)\right] \cdot Q^{-1}(v) \\
& =v^{(v-1) / q_{*}}\left(v \ln v^{1 / q_{*}}+v+1\right) v^{1 / q / q_{*}} \\
& =v^{1 / P}\left[\ln v+(v+1) p_{*}\right] /\left(p_{*} q_{*}\right) .
\end{aligned}
$$

We now observe that the second derivative of $P^{-1}$ is negative for large enough positive values of the argument, that

$$
\lim _{u \rightarrow \infty} u P^{\prime}(u) / P(u)=\lim _{v \rightarrow \infty} P^{-1}(v) / v\left(P^{-1}\right) \prime(v)=p,
$$

and that $1<p<n$. From these facts it follows that we may choose $u_{1}$ so that $P(u)$ is defined and convex for $u \geq u_{1}$, and that

$$
\rho=u_{1} P^{\prime}\left(u_{1}\right) / P\left(u_{1}\right)
$$

satisfies $1<\rho<n$. We can now complete the definition of $P$ as an $N$-function by using (4.4) to define $P(u)$ for $|u| \geq u_{1}$, and setting

$$
\left.P(u)=P\left(u_{1}\right)(|u|) / u_{1}\right)^{\rho} \quad \text { for } 0 \leq|u| \leq u_{1} \text {. }
$$




\section{Grahame Hardy}

Finally, because $1<\rho<n$, we find from (4.5) that

$$
\int_{0}^{1} P^{-1}(t) \cdot t^{-1-1 / n} d t<\infty
$$

and because $1<p<n,(4.4)$ and an obvious comparison argument shows that

$$
\lim _{s \rightarrow \infty} \int^{s} P^{-1}(t) \cdot t^{-1-1 / n} d t=\infty .
$$

Thus $M, P, Q$ and $R$ satisfy all the conditions on them which occur in Theorem 3.1 .

\section{References}

[1] Robert A. Adams, Sobolev spaces, Pure and Applied Mathematics, 65. Academic Press (Harcourt Brace Jovanovich), New York, San Francisco, London, 1975

[2] Thomas K. Donaldson and Neil s. Trudinger, "Orlicz-Sobolev spaces and imbedding theorems", J. Funct. Anal. 8 (1971), 52-75.

[3] Jean-Pierre Gossez, "Non linear elliptic boundary problems for equations with rapidly (or slowly) increasing coefficients", Trans. Amer. Math. Soc. 190 (1974), 163-205.

[4] Grahame Hardy, "Extensions of theorems of Gagliardo and Marcus and Mizel to Orlicz spaces", Bull. Austral. Math. Soc. 23 (1981), 121-138.

[5] M. A. Krasnosel'skii and Ya.B. Rutickii, Convex functions and Orlicz spaces (translated from the first Russian edition by Leo F. Boron. Noordhoff, Groningen, 1961).

[6] M. Marcus and V. J. Mizel, "Absolute continuity on tracks and mappings of Sobolev spaces", Arch. Rational Mech. Anal. 45 (1972), 294-320. 
Nemitsky operators between Orlicz-Sobolev spaces

[7] M. Marcus and V.J. Mizel, "Nemitsky operators on Sobolev spaces", Arch. Rational Mech. Anal. 51 (1973), 347-370.

Division of External Studies,

University of Queensland,

St. Lucia,

Queensland 4067,

Australia. 